\title{
INFORMAÇÕES CONTIDAS NOS MANUAIS DE TESTES DE PERSONALIDADE
}

\author{
Ana Paula Porto Noronha* \\ Fernanda Andrade Freitas \\ Fernanda Argentini Sartori" \\ Fernanda Ottati"
}

\begin{abstract}
RESUMO. O objetivo da pesquisa foi avaliar testes de personalidade no que se refere à presença ou ausência de informações nos manuais de testes de personalidade. Foram consultados vinte e dois desses manuais com o objetivo de verificar se continham as seguintes informações: nome do autor, editor e data de publicação do manual em português, variável medida, áreas de aplicação, formato e número dos itens, suporte, correção, transformação da pontuação, escala utilizada, tempo de aplicação, documentação fornecida e bibliografia utilizada. Os resultados indicaram que as informações autor e editor estavam presentes em todos os testes e dez dos instrumentos consultados apresentaram todas as informações analisadas nos seus respectivos manuais.
\end{abstract}

Palavras-chaves: testes psicológicos, manuais de testes, avaliação psicológica.

\section{INFORMATION AVAILABLE IN THE HANDBOOKS OF PERSONALITY TESTS}

\begin{abstract}
The research had as objective to evaluate personality tests in which it relates to the presence or absence of information in the respective manuals. Twenty two manuals of tests had been consulted with the objective of verifying if they had the following information: name of the author, publisher and date of the manual publication in Portuguese, the construct, publication areas, shape and number of the items, support, correction, changing of punctuation, used scales, application time, supplied documentation and used bibliography. The results have indicated that the information about the author and publisher were written in all the tests and that ten of the consulted instruments have presented all the information analyzed in its respective manuals.
\end{abstract}

Key words: psychological tests, manual of tests, psychological evaluation.

\section{INTRODUÇÃO}

Os testes psicológicos são instrumentos úteis ao psicólogo, exclusivos de sua prática profissional, e podem colaborar quando do processo de avaliação psicológica, no que diz respeito à coleta e interpretação de dados. O número de instrumentos não é pequeno, sobretudo nos países do Primeiro Mundo, onde o desenvolvimento da pesquisa científica já acumulou grandes conquistas. No Brasil, tenta-se superar as dificuldades relativas ao desenvolvimento de pesquisas e, embora não se tenha uma grande variedade de bons testes para avaliar vários construtos, validados para nossa realidade, os pesquisadores vêm trabalhando na tentativa de resgatar a legitimidade e o status do instrumento padronizado.

Tendo sido alvo de críticas desde o seu início pela pouca precisão de seus resultados (Anastasi \& Urbina, 2000), os testes necessitam de pesquisas e de aprimoramento para que consolidem o seu verdadeiro valor, ou seja, o de auxiliar na prática do profissional de Psicologia. No entanto, muitas publicações no país não apresentam um mínimo de qualidade, ou seja, os testes muitas vezes não são adaptados nem padronizados para a realidade brasileira e não contêm as informações mínimas necessárias em seus manuais

Doutora em Psicologia: ciência e profissão pela PUC-Campinas; docente do Curso de Psicologia e do Programa de Estudos Pósgraduados em Psicologia, da Universidade São Francisco.

Endereço para correspondência: Rua Francisco Pinto Osório, 40, Jardim Morumbi, 13250-000, Itatiba-SP. E-mail: noronhafagundes@netwave.com.br

\# Bolsista de Iniciação Científica (PROBAIC/USF).

II Aluna do Curso de Psicologia, da Universidade São Francisco. 
(Noronha, Sbardelini \& Sartori, 2001; Noronha, Sartori, Freitas \& Ottati, 2001; Noronha, 2001).

Segundo Andriola (1995), alguns testes psicológicos passaram por estudos, mas há muito tempo, quando não havia o auxílio dos computadores; portanto, faz-se necessário que novos estudos sejam realizados, com o objetivo de minimizar os erros resultantes de precárias análises estatísticas. Além disso, outro problema comumente encontrado em relação ao uso dos instrumentos psicológicos diz respeito a serem os testes somente traduzidos, faltando estudos prévios justificativos da validade de seu uso (Azevedo, Almeida, Pasquali \& Veiga, 1996). Embora essa seja uma questão antiga, que fez parte de manuais clássicos da avaliação psicológica no Brasil (Van Kolch, 1981; Anastasi, 1977), ainda é possível encontrar instrumentos traduzidos de outros países que não foram submetidos a nenhum estudo de padronização. Tal questão é séria, pois coloca em questionamento a confiabilidade dos instrumentos e do processo de avaliação como um todo.

De acordo com Cronbach (1996), os profissionais, ao escolherem os testes que irão utilizar ou comprar, devem consultar alguns manuais, como o Mental Measurements Yearbooks e o Test Critiques, tendo em vista que essas publicações poderão oferecer dados importantes sobre as características do instrumento. Essas revisões visam a melhorar a qualidade dos testes publicados, já que trazem várias informações sobre eles, e conseqüentemente tendem a colaborar para que os processos de coleta de dados ofereçam resultados mais seguros.

No Brasil, ainda não há esse tipo de material sistematizado que ofereça ao psicólogo informações pertinentes à construção, à aplicação e às qualidades psicométricas dos instrumentos, embora existam iniciativas isolados nesse sentido. Cunha e cols. (2000) realizaram a análise crítica de alguns testes psicológicos, oferecendo dados como o histórico, a aplicação e bibliografia para consulta. A inexistência de um levantamento oficial dos testes disponíveis no Brasil dificulta o caminhar das pesquisas, uma vez que impede um delineamento metodológico mais complexo e preciso. Por ora, a única maneira de reunir os instrumentos disponíveis no mercado psicológico brasileiro é consultar as diferentes editoras brasileiras.

Para Salvia e Ysseldyke (1991), o psicólogo deve estudar cuidadosamente os manuais de testes e, segundo determinação do comitê da American Psychological Association, da American Educational Research Association e do National Council on Measurement in Education, qualquer manual deve oferecer ao usuário as informações importantes para que a escolha do material esteja em concordância com os objetivos e necessidades da medida. Esta é uma tarefa a ser desenvolvida pelos psicólogos, na tentativa de obter avaliações mais seguras e precisas. No Brasil, recentemente o CFP (Conselho Federal de Psicologia, 2001) publicou a Resolução $n^{\circ} 25 / 2001$, que define teste como um método de avaliação privativo do psicólogo e regulamenta sua elaboração, comercialização e uso. Ela determina que os manuais dos testes contenham informações acerca da fundamentação teórica dos instrumentos da aplicação, avaliação e interpretação e da literatura científica relacionada ao instrumento, indicando os meios para a sua obtenção.

\section{TESTES DE PERSONALIDADE}

Estudos realizados demonstram que os testes de personalidade estão presentes na prática do psicólogo (Noronha, 2000; Alchieri \& Scheffel, 2000) e representam a maior parcela de instrumentos disponíveis no mercado profissional. Isto se justifica pelo fato de avaliações da personalidade serem imprescindíveis em diversos contextos de atuação do psicólogo.

Em relação às técnicas projetivas, o número de pesquisas é muito pequeno e quase escasso no caso de crianças (Cunha e cols., 2000), mas apesar disso alguns autores acreditam que sua popularidade ainda se preserva em função do refinamento de seus sistemas de avaliação e de interpretação (Hutz \& Bandeira, 1993). Não parece cabível que técnicas projetivas se transformem em psicométricas, mas em contrapartida, pesquisas de várias naturezas são sempre apropriadas para que tais técnicas não sejam recebidas como meros estímulos subjetivos. Alves (1997) justifica que os testes projetivos têm ampla aplicação para investigação e diagnóstico da personalidade, sendo essenciais para a realização da avaliação psicológica.

Já no que se relaciona aos inventários de personalidade, Piotrowski (2000) realizou um estudo a fim de verificar a popularidade dos Inventários de Avaliação da Personalidade e chegou à conclusão de que eles estão entre os mais utilizados na avaliação da personalidade, pois podem oferecer dados importantes para a prática clínica. Tal estudo pode ser complementado pelo de Meehl (2000), que afirma que os testes de personalidade em forma de questionário oferecem vantagens em relação aos não estruturados, tendo-se em vista que seus itens são selecionados empiricamente. 
É possível encontrar na literatura internacional diversos estudos que versam sobre as técnicas de avaliação da personalidade. Weiner (1999) discutiu a perspectiva contemporânea da avaliação do Rorschach, considerando o respeito científico e profissional que o instrumento possui, e Shafer (1999) investigou a relação entre o "Big Five", as Escalas de Personalidade de Comrey e o Teste de Personalidade "Howarth".

Já na literatura nacional, o que pode ser observado é que há uma escassez de estudos a respeito dos instrumentos de avaliação da personalidade. Alguns dos disponíveis versam sobre correlações de resultados dos testes de personalidade com alguns outros aspectos, como ciúmes, sexualidade e cultura, mas dificilmente se encontram estudos e pesquisas a respeito da validade dos instrumentos em si (Oliveira, 2001).

Segundo Pasquali (1999), há uma crescente demanda de instrumentos de avaliação, mas ela não está acompanhada de um desenvolvimento de tais instrumentos por parte dos pesquisadores. Acreditase inclusive que a Resolução $n^{\circ} 25 / 2001$ do CFP contribua para que novos estudos sejam desenvolvidos a fim de se preencherem os critérios estabelecidos. Mas é preciso pesquisar, mesmo que os estudos tenham objetivos modestos e parciais, que garantam apenas uma pequena parte das necessidades da área.

Levando em consideração a urgente necessidade de pesquisas na área de avaliação psicológica, o presente estudo focará os Testes de Personalidade, com o objetivo de analisar os manuais destes testes em relação aos seguintes aspectos: autor e instrução, editor, variável medida, tempo de aplicação, formato dos itens, suporte, correção, transformação da pontuação, documentação e referência bibliográfica.

\section{MÉTODO}

\section{Instrumentos consultados}

Para o levantamento de dados da presente pesquisa, foram consultados 22 manuais de testes psicológicos de avaliação da personalidade. Não foram analisados todos os instrumentos de avaliação disponíveis no mercado brasileiro, porque este não era o objetivo do estudo, mas certamente a amostra analisada é representante da maior parte, embora a ausência de indicadores oficiais nacionais sobre os testes psicológicos no Brasil, como o Mental Measurements Yearbooks, de alguma forma, dificulte este tipo de levantamento.

Vale destacar que os parênteses que se seguem à relação dos instrumentos indicam o autor original, a editora de publicação no Brasil e a data do manual avaliado. São eles:

- Cornell Index (Weider, Wolf, Brodman, Mittelman \& Wechsler, CEPA, sem data);

- 16 PF- $5^{a}$ edição (Cattell, Cattell \& Cattell, CEPA, 1999);

- Inventário Multifásico Minnesota de Personalidade- MMPI (Hathaway \& McKinley, CEPA, sem data);

- Pirâmides Coloridas de Pfister (Heiss \& Hiltmann, CEPA, 1976);

- Questionário de Personalidade Dadahie (Andrade, Moraes \& Wendel, CEPA, sem data);

- Testes das Cores (Braga, CEPA, 1978);

- Teste Projetivo Ômega- TPO (Villas Boas Filho, CEPA, 1967);

- Escala de Personalidade de Comrey (Comrey, Vetor, 1997);

- Psicodiagnóstico Miocinético- PMK (Mira y López, Vetor, 1987);

- Questionário de Avaliação Tipológica - QUATI (Zacharias, Vetor, 1994);

- Teste Caracterológico- TCO (Minicucci, Vetor, 1996);

- Teste das Pirâmides de Cores (Heiss \& Halder, Vetor, 1971);

- Teste Palográfico (Milá, Vetor, 1976);

- Inventário Fatorial de Personalidade- IFP (Edwards, Casa do Psicólogo, 1997);

- Teste de Wartegg (Wartegg, Casa do Psicólogo, 1993);

- Teste de Apercepção Infantil- CAT A (Bellak \& Bellak, Mestre Jou, 1981);

- Teste de Apercepção Temática - TAT (Murray, Mestre Jou, 1973);

- Teste de Apercepção Infantil - CAT H (Bellak \& Bellak, Psy, 1992);

- Teste de Apercepção para idosos - SAT (Bellak \& Bellak, Psy, 1992);

- Suplemento para Teste de Apercepção InfantilCAT S (Bellak \& Bellak, Psy, 1992);

- Teste de Rorschach (Rorschach, Manole, 1997);

- Teste das Fábulas (Düss, CETEPP, 1993).

\section{CRITÉRIOS DE AVALIAÇÃO}

Para a realização da pesquisa as autoras se basearam no questionário proposto por Prieto \& Muñiz (2000), que procuraram originalmente avaliar os testes usados na 
Espanha. Ele tem como proposta a avaliação de vários aspectos e, dentre eles, foram selecionados no presente estudo aqueles relacionados aos dados de identificação e dados de aplicação e de avaliação, a saber: autor, editor, data da publicação, variável (classificação geral da variável do constructo que se pretende medir), áreas de aplicação (se há especificação no manual relativa às áreas para as quais o teste se destina), formato dos itens (resposta livre, respostas dicotômicas, eleição múltipla_, tipo Likert, adjetivos bipolares, outros), número de itens, suporte (administração oral, lápis e papel, manipulativo, informatizado, outros), correção (crivo, leitura ótica, realizado por aplicador, com a ajuda de experts, outros), transformação da pontuação (característica não aplicável ao instrumento, normatizada, não normatizada), tempo requerido para aplicação, documentação oferecida (manual, livros ou artigos complementares, disquetes, $\mathrm{CD}$, outros) e bibliografia básica acerca do teste.

De acordo com os objetivos desse trabalho, não foram considerados outros possíveis elementos de análise, como o tipo de documentação oferecida, a quantidade de referências fornecida_ou a área de aplicação do teste. Serviu como elemento_ de análise apenas a informação que constava no manual consultado, ou seja, a presença ou ausência de algum tipo de informação.

\section{ANÁLISE DOS RESULTADOS:}

Após a coleta, os dados obtidos foram organizados em três tabelas, e o critério para a avaliação dos testes foi a presença ou a ausência das informações (dados de identificação, de aplicação e de avaliação) contidas nos respectivos manuais. Ao final de cada Tabela é possível encontrar o DP e a média relacionados aos aspectos avaliados.

No caso da presença da informação o teste recebeu um ponto, e na ausência, não recebeu pontuação. Uma segunda análise foi a aplicação do teste de qui-quadrado para avaliar se eram ou não homogêneas as respostas dos sujeitos, trabalhando-se com o nível de significância de 0,05, amplamente aceito na área da ciências humanas.

\section{RESULTADOS}

A Tabela I apresenta os resultados relativos aos dados de identificação do instrumento, ou seja, autor, editor e data de publicação; a pontuação máxima nesta avaliação é três. Em todos os manuais consultados havia o (s) nome (s) do (s) autor (es) e da editora. Em relação à data, foi possível encontrar 22,73\% (N=5) dos instrumentos sem tal informação, o que significa dizer que $77,27 \%$ dos instrumentos consultados apresentaram todos os dados de identificação, enquanto o restante não apresentou uma das informações (editor ou data).

Tabela 1 - Dados de identificação dos instrumentos.

\begin{tabular}{lccc}
\hline Nome do teste & Autor & Editor & data \\
\hline Cornell Index & 1 & 1 & 0 \\
16 PF- 5a edição & 1 & 1 & 0 \\
Escala de Personalidade de Comrey & 1 & 1 & 1 \\
Inventário Fatorial de Personalidade- IFP & 1 & 1 & 1 \\
Inventário Multifásico Minesota de Personalidade- MMPI & 1 & 1 & 0 \\
Pirâmides Coloridas de Pfister & 1 & 1 & 1 \\
Psicodiagnóstico Miocinético- PMK & 1 & 1 & 0 \\
Questionário de Avaliação Tipológica- QUATI & 1 & 1 & 1 \\
Questionário de Personalidade Dadahie & 1 & 1 & 0 \\
Teste de Rorschach & 1 & 1 & 1 \\
Suplemento para teste de apercepção infantil- CATS & 1 & 1 & 1 \\
Teste Caracterológico- TCO & 1 & 1 & 1 \\
Testes das Cores & 1 & 1 & 1 \\
Teste das Fábulas & 1 & 1 & 1 \\
Teste das Pirâmides de Cores & 1 & 1 & 1 \\
Teste de Apercepção Infantil- CAT A & 1 & 1 & 1 \\
Teste de Apercepção Infantil- CAT H & 1 & 1 & 1 \\
Teste de Apercepção para idosos- SAT & 1 & 1 & 1 \\
Teste de Apercepção Temática- TAT & 1 & 1 & 1 \\
Teste Palográfico & 1 & 1 & 1 \\
Teste Projetivo Omega- TPO & 1 & 1 & 1 \\
Teste de Wartegg & 1 & 1 & 1 \\
Média & 1 & 1 & 0,77 \\
DP & 0 & 0 & 0,43 \\
\hline
\end{tabular}

A Tabela 1 apresenta os dados de aplicação e de avaliação dos instrumentos. Os aspectos avaliados foram: variáveis medidas, forma de aplicação, formato dos itens, número de itens, suporte, correção, pontuação, tempo de aplicação, documentação aportada e referências bibliográficas que constam do manual. A pontuação máxima nesta avaliação é dez.

Todos os instrumentos consultados apresentaram seis $(60 \%)$ das dez informações, a saber: descrição da variável medida, formato dos itens, número de itens, suporte, correção e pontuação. Dentre os demais aspectos - forma de aplicação, tempo, documentação fornecida e bibliografia citada - o pior avaliado foi tempo requerido para aplicação. Tal constatação é importante, uma vez que nem sempre aparece especificado no manual quanto tempo será necessário para a aplicação do instrumento. Essa informação, bem como o tempo necessário para avaliar o instrumento, devem configurar-se como critérios de escolha ou de rejeição dos instrumentos de avaliação.

Tomando-se como referência a análise de cada teste, vale ressaltar que treze receberam pontuação máxima nesta avaliação. Os instrumentos que receberam a menor pontuação foram o C.A.T. - humano e o Questionário de Personalidade Dadahie. 
Tabela 2 - Dados de aplicação e de avaliação.

\begin{tabular}{|c|c|c|c|c|c|c|c|c|c|c|}
\hline Nome do teste & Variável & Aplicaç & Itens forma & Itens & Suporte & Correção & Pontos & tempo & Docum & Biblio \\
\hline Cornell Index & 1 & 1 & 1 & 1 & 1 & 1 & 1 & 1 & 1 & 1 \\
\hline 16 PF- $5^{\mathrm{a}}$ edição & 1 & 1 & 1 & 1 & 1 & 1 & 1 & 1 & 1 & 1 \\
\hline Escala de Personalidade de Comrey & 1 & 1 & 1 & 1 & 1 & 1 & 1 & 0 & 1 & 1 \\
\hline Inventário Fatorial de Personalidade- IFP & 1 & 1 & 1 & 1 & 1 & 1 & 1 & 1 & 1 & 1 \\
\hline Inventário Multifásico Minesota de Personalidade- MMPI & 1 & 1 & 1 & 1 & 1 & 1 & 1 & 0 & 1 & 1 \\
\hline Pirâmides Coloridas de Pfister(*) & 1 & 1 & 1 & 1 & 1 & 1 & 1 & 1 & 1 & 1 \\
\hline Psicodiagnóstico Miocinético- PMK & 1 & 1 & 1 & 1 & 1 & 1 & 1 & 1 & 1 & 1 \\
\hline Questionário de Avaliação Tipológica- QUATI & 1 & 1 & 1 & 0 & 1 & 1 & 1 & 1 & 1 & 1 \\
\hline Questionário de Personalidade Dadahie & 1 & 0 & 1 & 1 & 1 & 1 & 1 & 1 & 1 & 0 \\
\hline Teste de Rorschach & 1 & 1 & 1 & 1 & 1 & 1 & 1 & 1 & 1 & 1 \\
\hline Suplemento para teste de apercepção infantil- CATS & 1 & 1 & 1 & 1 & 1 & 1 & 1 & 1 & 1 & 1 \\
\hline Teste Caracterológico- TCO & 1 & 1 & 1 & 1 & 1 & 1 & 1 & 1 & 1 & 1 \\
\hline Testes das Cores & 1 & 1 & 1 & 1 & 1 & 1 & 1 & 0 & 1 & 1 \\
\hline Teste das Fábulas & 1 & 1 & 1 & 1 & 1 & 1 & 1 & 1 & 1 & 1 \\
\hline Teste das Pirâmides de Cores & 1 & 1 & 1 & 1 & 1 & 1 & 1 & 1 & 1 & 1 \\
\hline Teste de Apercepção Infantil- CAT A & 1 & 1 & 1 & 1 & 1 & 1 & 1 & 0 & 1 & 1 \\
\hline Teste de Apercepção Infantil- CAT H & 1 & 0 & 1 & 1 & 1 & 1 & 1 & 0 & 1 & 1 \\
\hline Teste de Apercepção para idosos- SAT & 1 & 1 & 1 & 1 & 1 & 1 & 1 & 1 & 1 & 1 \\
\hline Teste de Apercepção Temática- TAT & 1 & 1 & 1 & 1 & 1 & 1 & 1 & 1 & 1 & 0 \\
\hline Teste Palográfico & 1 & 1 & 1 & 1 & 1 & 1 & 1 & 1 & 1 & 1 \\
\hline Teste Projetivo Omega- TPO & 1 & 1 & 1 & 1 & 1 & 1 & 1 & 1 & 1 & 0 \\
\hline Teste de Wartegg & 1 & 1 & 1 & 1 & 1 & 1 & 1 & 1 & 1 & 1 \\
\hline Média & 1 & 0,91 & 1 & 0,95 & 1 & 1 & 1 & 0,77 & 1 & 0,86 \\
\hline DP & 0 & 0,29 & 0 & 0,21 & 0 & 0 & 0 & 0,43 & 0 & 0,35 \\
\hline
\end{tabular}

Na Tabela 3 é possível encontrar a pontuação máxima recebida por cada instrumento nos critérios avaliados por este estudo. Na segunda coluna estão as notas referentes aos dados de identificação; na terceira, as notas referentes aos dados de aplicação e de avaliação e na última, a soma das duas anteriores. A pontuação máxima na coluna um é 3 , na segunda coluna, $10 \mathrm{e}$, no total, 13.

Tabela 3 - Pontuação geral dos testes.

\begin{tabular}{lccc}
\hline Nome do Teste & I & II & Total \\
\hline Cornell Index & 2 & 10 & 12 \\
16 PF- 5 edição & 2 & 10 & 12 \\
Escala de Personalidade de Comrey & 3 & 9 & 12 \\
Inventário Fatorial de Personalidade- IFP & 3 & 10 & 13 \\
Inventário Multifásico Minesota de Personalidade-MMPI & 2 & 9 & 11 \\
Pirâmides Coloridas de Pfister & 3 & 10 & 13 \\
Psicodiagnóstico Miocinético- PMK & 2 & 10 & 12 \\
Questionário de Avaliação Tipológica- QUATI & 3 & 9 & 12 \\
Questionário de Personalidade Dadahie & 2 & 8 & 10 \\
Teste de Rorschach & 3 & 10 & 13 \\
Suplemento para teste de apercepção infantil- CATS & 3 & 10 & 13 \\
Teste Caracterológico- TCO & 3 & 10 & 13 \\
Testes das Cores & 3 & 9 & 12 \\
Teste das Fábulas & 3 & 10 & 13 \\
Teste das Pirâmides de cores & 3 & 10 & 13 \\
Teste de Apercepção Infantil- CAT A & 3 & 9 & 12 \\
Teste de Apercepção Infantil- CAT H & 3 & 8 & 11 \\
Teste de Apercepção para idosos- SAT & 3 & 10 & 13 \\
Teste de Apercepção Temática- TAT & 3 & 9 & 12 \\
Teste Palográfico & 3 & 10 & 13 \\
Teste Projetivo Omega- TPO & 3 & 9 & 12 \\
Teste de Wartegg & 3 & 10 & 13 \\
Média & 2,77 & 9,05 & \\
DP & 0,43 & 2,13 & \\
\hline
\end{tabular}

A partir dos dados obtidos, pôde-se observar que dez instrumentos receberam a pontuação máxima no que se refere aos dados de identificação, aplicação e avaliação, sendo que os demais instrumentos deixaram de apresentar uma, ou no máximo 3 das informações avaliadas. $O$ pior resultado foi encontrado no Questionário de Personalidade Dadahie.

$\mathrm{Na}$ análise das médias das duas colunas da Tabela III (dados de identificação e dados de aplicação e de avaliação), fica claro que os dados de identificação receberam uma avaliação um pouco melhor neste estudo, em detrimento dos demais aspectos avaliados, considerando-se que, proporcionalmente, a primeira coluna atingiu $92,3 \%$ da pontuação máxima, enquanto a segunda, $88,8 \%$.

Em estudo desenvolvido pelas mesmas autoras, realizado com testes de inteligência, a fim de se verificar a presença ou ausência destas mesmas informações, encontraram-se resultados diferentes, ou seja, a melhor avaliação foi a dos dados de aplicação e de avaliação (Noronha, Freitas, Sartori \& Ottati, 2001).

$\mathrm{Na}$ análise estatística foi utilizado o teste de quiquadrado para verificar a homogeneidade da distribuição ( $X^{2} \mathrm{c}=32,671$; n.g.l. 21; n. sign. 0,05). Os valores encontrados foram 1,609 para os dados de identificação e de 1,1606 para os dados de aplicação e de avaliação, portanto não significativas, podendo-se dizer que os itens avaliados se distribuem igualmente nos instrumentos estudados. A inserção desse tipo de análise se justifica pela necessidade de verificar se a distribuição dos itens se manifesta diferentemente nos instrumentos consultados, o que não ocorreu. 


\section{DISCUSSÃO}

Vale ressaltar que, embora os resultados desse estudo não tenham gerado surpresa em relação ao assunto, trabalhos desta natureza são necessários, tendo-se em vista que revelam e, de alguma forma, comprovam o que há algum tempo os profissionais da área vêm denunciando: os instrumentos psicológicos necessitam de mais pesquisas que objetivem resgatar seu status. Embora os resultados não tenham sido completamente alentadores, uma vez que retrataram certo descuido em relação a determinados testes de personalidade, mais da metade dos consultados apresentaram em seus manuais todas as informações abordadas no presente estudo. No entanto, os itens avaliados nos manuais referem-se aos aspectos informativos necessários para o uso adequado do material, e a ausência de quailquer um deles pode inibir uma compreensão mais abrangente do instrumento em questão.

Não parece mais novidade que testes sejam publicados sem que os manuais apresentem as informações necessárias para uma utilização adequada e satisfatória. Manuais sem data, com a ausência do nome do autor ou do editor, sem a consistente fundamentação teórica, sem as informações das pesquisas de padronização, ou ainda, sem informações sobre validade e precisão, infelizmente não são incomuns.

Autores já enfatizaram a importância que se deve atribuir ao processo de construção, tradução, adaptação ou padronização de testes psicológicos (Oakland, 1999; Adanéz, 1999; ITC, 2001; Salvia \& Ysseldyke, 1991 e Cunha e cols., 2000). Embora os trabalhos tenham focos diferentes, eles têm em comum o alerta a respeito do cuidado que pesquisadores e usuários de teste devem despender aos instrumentos em todas as suas etapas, desde a construção até a utilização. A Resolução no 25/2001 do CFP (CFP, 2001) vem corroborar a necessidade de que instrumentos apresentem em seus manuais informações técnicas relevantes para a escolha e o uso adequado dos testes psicológicos, de foram a garantir bons resultados. As faltas não são poucas no que se refere à construção de instrumentos.

Vale destacar que os dados deste estudo são o resultado da análise de instrumentos que se destinam à avaliação da personalidade; instrumentos estes muito questionados, pela falta de estudos relativos às suas qualidades psicométricas (Halperin \& McKay, 1998).

Há muita controvérsia em relação a este tema. Por um lado, acredita-se que testes de personalidade têm características que os diferenciam perante outros instrumentos. Em outra perspectiva, sabe-se que determinados materiais, apoiando-se nesta crença, ou seja, de que testes de personalidade têm características específicas, acabam não apresentando certos cuidados necessários à construção de instrumentos científicos (Alves, 1997). O resultado é catastrófico, uma vez que é possível encontrar a absoluta falta de qualidade em materiais psicológicos em geral.

Os testes de personalidade $\mathrm{e}$ as técnicas projetivas, em especial, possuem características específicas, que os diferenciam dos demais. Embora essa diferença não possa ser ignorada, Anzieu (1979) aponta que deve haver um remanejamento dos conceitos de medida, no caso desse tipo de instrumental. $\mathrm{O}$ autor propõe uma discussão diferenciada para a sensibilidade, para a validade e para a precisão. Tal colocação pode ser corroborada por McFarlane e Tuddenham (1978), ao afirmarem que o dilema da pesquisa sobre testes projetivos centra-se na dificuldade de integração de valores, métodos e contribuições de pesquisas dos três princípios de psicologia: experimental, estatístico e clínico. Para os autores, o avanço do material depende da integração dessas contribuições. E a ausência de integração, de alguma forma, pode justificar a distribuição relativamente semelhante da pontuação nos instrumentos consultados neste estudo, ou seja, os instrumentos têm esta falha em comum.

Apesar das críticas sofridas pelos testes, os de personalidade, em especial, são considerados os mais requisitados pela comunidade psicológica. Isto fica evidenciado em estudos nacionais e internacionais. Entre os internacionais, destaca-se o de Archer e Newson (2000), que apresentou a relação dos instrumentos usados na clínica com adolescentes, entre os quais é possível encontrar o Rorschach, o MMPI e o T.A.T. Outros estudos nacionais já foram destacados na introdução deste trabalho (Noronha, 2000; Alchieri \& Scheffel, 2000).

Estudos como o de Meyer e cols. (2001) confirmam que a avaliação é uma complicada atividade que requer compreensão sofisticada da personalidade e da psicopatologia, entre outros domínios; conhecimento de mensuração psicológica, estatística e métodos de pesquisa; o reconhecimento de que diferentes métodos de avaliação produzem informações qualitativamente diferentes; e ainda compreensão de que os métodos são limitados.

À medida que a comunidade psicológica reconhecer tais limitações e buscar a superação dos obstáculos, acredita-se que melhores testes psicológicos serão criados e os já existentes passarão por estudos e serão aperfeiçoados, a fim de facilitar o exercício profissional na área de avaliação psicológica. Para tanto, deve haver um esforço 
conjunto entre pesquisadores, profissionais, editores e os Conselhos Federal e Regionais de Psicologia.

Sugere-se que outros estudos sejam desenvolvidos com outros instrumentos ou que os citados sejam submetidos a outros tipos de análise, como por exemplo, a avaliação dos estudos de validade e de precisão.

\section{REFERÊNCIAS BIBLIOGRÁFICAS}

Adánez, G. P. (1999). Procedimientos de Construccion y Analisis de Tests Psicometricos. In S. M. Wechsler \& R. S. L. Guzzo (Orgs.). Avaliação Psicológica - perspectiva internacional. São Paulo: Casa do Psicólogo, p. 57-100

Alchieri, J. C. \& Scheffel, M. (2000). Indicadores da Produção Científica Brasileira em Avaliação Psicológica: resultados da elaboração de uma base de dados dos artigos publicados em periódicos brasileiros de 1930 a 1999. V Encontro Mineiro de Avaliação Psicológica - teorização e prática, VIII Conferência Internacional Avaliação Psicológica - formas e contextos, PUC Minas, Belo Horizonte/MG, p. 99

Alves, I. C. B. (1997). As técnicas Projetivas no Psicodiagnóstico e sua Função na Psicoterapia. Anais do I Encontro sobre Psicologia Clínica. Universidade Mackenzie, São Paulo, p. 9-14

Anastasi, A. (1977). Testes Psicológicos. Porto alegre: Artmed

Anastasi, A. \& Urbina, S. (2000). Testagem Psicológica. Porto Alegre: Artmed

Andriola, W. B. (1995). Os Testes Psicológicos no Brasil: problemas, pesquisas e perspectivas para o futuro. Em L. S. Almeida \& I. S. Ribeiro (Org.). A valiação Psicológica - formas e contextos, vol. 3, APPORT, Braga, p. 77-82

Anzieu, D. (1979). Os Métodos Projetivos. Rio de Janeiro: Campus ( $2^{\mathrm{a}}$ edição)

Archer, R. P. \& Newson, C. R. (2000) - Psychological Test Usage with Adolescent Clients: survey update. Assessment, 7(3): 227-235

Azevedo, M. M.; Almeida, L. S.; Pasquali, L. \& Veiga, H. M. S. (1996). Utilização dos Testes Psicológicos no Brasil: dados de estudo preliminar em Brasília. Em L. S. Almeida; S. Araújo, M. M. Gonçalvez; C. Machado \& M. R. Simões (Orgs.). Avaliação Psicológica - formas e contextos, vol. IV, APPORT / Braga, p. 213-220

CFP (2001). Resolução $n^{0}$ 25/2001. [Disponível: www.pol.org.br]. Acessado em 01/04/2002.

Cronbach, L. J. (1996). Fundamentos da Testagem Psicológica. Porto Alegre: Artes Médicas, $5^{a}$ edição

Cunha, J. A. e colaboradores (2000). Psicodiagnóstico V. Porto alegre: Artes Médicas

Halperin, J. M. \& McKay, K. E. (1998). Psychological Testing for Child and Adolescent Psychiatrists: a review of the past 10 years. Journal of the American Academy of Child and Adolescent Psychiatry, 37(6): 575-584

Hutz, C. S. \& Bandeira, D. R. (1993). Tendências Contemporâneas no Uso de Testes: uma análise da literatura brasileira e internacional. Psicologia: Reflexão e Crítica, 6(1/2): 85-108

ITC - Comisión Internacional de Tests (2001). Diretrices Internacionales para el Uso de los Tests. Colegio Oficial de
Psicólogos. [Disponível em: http://www.cop.es/tests]. Acessado em: 04/05/2001

McFarlane, J. W. \& Tuddenham, R. D. (1978). Dificuldades para a Validação das Técnicas Projetivas. Em H. H. Anderson \& G. L. Anderson - Técnicas Projetivas do Diagnóstico Psicológico. São Paulo: Mestre Jou, p. 41-68

Meehl, P. E. (2000). The Dynamics of "Structured" Personality Tests. Journal of Clinical Psychology, 56(3): 367-373

Meyer, G. J.; Finn, S. E.; Eyde, L. D.; Kay, G. G.; Moreland, K. L.; Dies, R. R.; Eisman, E. J.; Kubiszyn, T. W. \& Reed, G. M. (2001). Psychological Testing and Psychological Assessment - a review of evidenced and issues. American Psychologist, 56(2): 128-165

Noronha, A. P. P. (2000). Avaliação Psicológica e os Instrumentos Psicológicos mais utilizados por Psicólogos. Anais do $V$ Encontro Mineiro de Avaliação Psicológica - teorização $e$ prática; VIII Conferência Internacional Avaliação Psicológica - formas e contextos, PUC Minas, Belo Horizonte/MG, p. 67-68

Noronha, A. P. P.; Sartori, F. A.; Freitas, F. A. \& Ottati, F. (2001). Informações contidas nos manuais dos testes: um estudo com testes de inteligência. Psicologia em Estudo, 6(2): 101-106

Noronha, A. P. P.(2001). Análise de Coeficientes de Testes de Inteligência. Psico, 32(2): 73-86

Noronha, A. P. P.; Sartori, F. A.; Sbardelini, E. T. B. (2001). Análise da Qualidade de Testes de Inteligência Publicados no Brasil. Psico-USF, 6(2): 95 - 104.

Oakland, T. (1999). Developing Standardized Tests. In S. M. Wechsler \& R. S. L. Guzzo (Orgs.). Avaliação Psicológica: perspectiva internacional. São Paulo: Casa do Psicólogo, p. 101-118

Oliveira, M. F. (2001). Características de Personalidade em Indivíduos com Ciúme Patológico Submetidos ao Método de Rorschach. Anais do II Encontro de Iniciação Científica e V Mostra de Pós-graduação, Universidade Presbiteriana Mackenzie, São Paulo, p. 176

Pasquali, L. (1999). Instrumentos Psicológicos: manual prático de elaboração. Brasília: LabPAM / IBAPP, p. 13-25

Piotrowski, C. (2000). How Popular is the Personality Assessment Inventory in Practices and Training? Psychological Reports, 86(1): 65-66

Prieto, G. \& Muñiz, J. (2000). Um modelo para evaluar la calidad de los tests utilizados em España. [Disponível em: http://www.cop.es/tests], Acessado em: 04/12/2001

Salvia, J. \& Ysseldyke, J. (1991). Avaliação em Educação Especial e Corretiva. São Paulo: Ed. Manole

Shafer, A. B. (1999). Factor Analysis of Big Five Markers with the Comrey Personality Scales and the Howarth Personality Tests. Personality and Individual Differences, 26(5): 857-872

Van Kolch (1981). Técnicas do Exame Psicológico e suas Aplicações no Brasil. Petrópolis: Vozes.

Weiner, I. B. (1999). Contemporany Perspectives on Rorschach Assessment. European Journal of Psychological Assessment, 15(1): $78-86$

Recebido em 11/01/2002

Revisado em 10/06/2002 Aceito em 30/06/2002 
